Math. Model. Nat. Phenom.

Vol. 4, No. 1, 2009, pp. 183-194

DOI: $10.1051 / \mathrm{mmnp} / 20094109$

\title{
Singular Perturbations For Heart Image Segmentation Tracking
}

\author{
J. Pousin ${ }^{1}$ \\ Université de Lyon, Université Lyon 1; INSA de Lyon, F-69100; \\ Ecole Centrale de Lyon; CNRS, UMR5208, Institut Camille Jordan, \\ 20 Av. E. Einstein F-69100 Villeurbanne Cedex, France
}

\begin{abstract}
In this note we give a result of convergence when time goes to infinity for a quasi static linear elastic model, the elastic tensor of which vanishes at infinity. This method is applied to segmentation of medical images, and improves the 'elastic deformable template' model introduced previously.
\end{abstract}

Key words: images segmentation, linear elasticity, singular perturbations techniques.

AMS subject classification: 74B05, 35B75, 76M45, 74505

\section{Introduction}

Image segmentation is the process that extracts meaningful parts from images for further exploitation and quantification. Still here, there is no universal approach to the problem. It is clear however that a priori information related to the particular context is needed to particularize any generic segmentation method. Deformable models are classes of methods that has received a lot of attention in the past. They rely on a priori reference shape model of the structure to be extracted that is iteratively adapted to the image data at convergence. The adaptation process is usually driven by a force field issued from the image through the computation of the image gradient or gradient vector flow [2].

An a priori template (object) representing the heart is immersed into the image data and submitted to a force field which pulls the boundary of the object towards the image edges. This method

\footnotetext{
${ }^{1}$ E-mail: jerome.pousin@insa-lyon1.fr
} 
has several advantages but one drawback concerns the regularity of the displacement field and the smoothness of the final object boundary. As an alternative to classical geometrical curvature-based boundary regularization techniques, it is proposed in [5] to add boundary constraints modeling crudely some bio-mechanical properties of the heart. A simplified three-layer elastic model of the heart composed of a middle homogeneous isotropic layer and two surrounding thin layers of myocardial fibers with a directional structure is considered. The aim of this model was to mimic the elastic properties of the heart resulting from the fiber structure of the muscle oriented in the longitudinal direction. It is an efficient tool for image segmentation but not a complete myocardium model. It is proved in [5] that the fibrous layers can be replaced by boundary conditions on the middle layer whenever the thickness of the external layers tends to 0 . These conditions increase the stiffness of the boundary and smooth the displacement field at the interface of the elastic object by imposing preferential directions of deformation in the tangent space. In the context of bonded joint with soft material, similar contact relations and constitutive law models have been proposed in [6].

We are not going here to get into the details, but let us recall the elastic model. Let $\left(e_{i}\right)$ be the canonical orthonormal basis of the Euclidean space $\mathbb{R}^{3}$. In Cartesian coordinates the displacement field for any material point reads $u=u_{i} e_{i}$. The deformation is described by the Green-Lagrange strain tensor which is linearized under the small deformation assumption:

$$
e_{i j}(u)=\frac{1}{2}\left(\frac{\partial u_{i}}{\partial x_{j}}+\frac{\partial u_{j}}{\partial x_{i}}\right)
$$

If $\sigma$ denotes the stress tensor, the constitutive law or stress-strain relation for the homogeneous isotropic internal layer takes the form:

$$
\sigma(u)=\lambda \operatorname{trace}(e(u)) I+2 \mu e(u)
$$

where $\lambda$ and $\mu$ are the Lamé constants, and $I$ is the identity tensor. Equivalently, we have,

$$
e(u)=\frac{1+\nu}{E} \sigma(u)-\frac{\nu}{E} \operatorname{trace}(\sigma(u)) I,
$$

where $E$ is the Young modulus and $\nu$ the Poisson ratio. The following relations classically hold

$$
\frac{\nu}{E}=\frac{\lambda}{4 \mu(\lambda+\mu)}, \quad \frac{1+\nu}{E}=\frac{1}{2 \mu} .
$$

In Figure 1, on a segmentation of a mid-ventricular slice, the impact of the boundary conditions replacing the external fibrous layers is presented.

The asymptotic fibrous models increases the rigidity of the heart boundary which is favorable at the beginning of the segmentation process but not at the end. For the boundary of the heart to be correctly fitted, at the end of the segmentation process, the rigidity should be reduced, to allow for larger deformations. Basically, in 3D, the boundary of the heart is represented by the discontinuity surface of the gradient of the gray level function of the image. The GVF method, characterizes theses surfaces as being the zero of a function $\mathbf{f}(\mathbf{I}+\mathbf{u})$. 

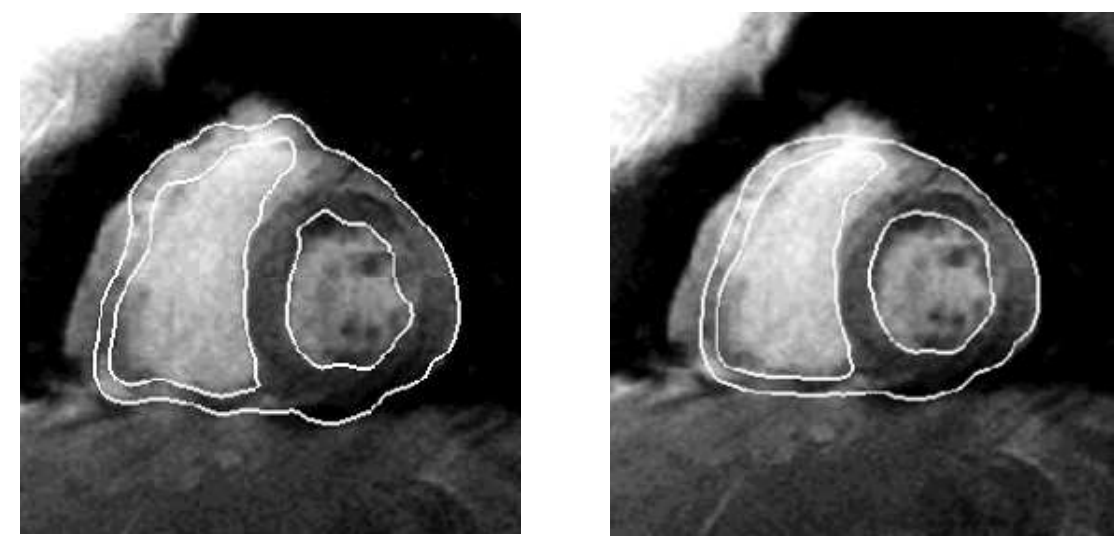

Figure 1: Impact of the boundary regularization on segmentation results for a mid-ventricular slice : without (left) and with (right) applied boundary conditions.

Whenever the iterative process converges, the cumulative forces, that is to say, the forces extracted from the image acting on the boundary of the deformed template (contours) should approach zero.

However, this is not achieved in classical deformable model since the work of the forces $\mathbf{f}$ are compensated by the internal elastic energy [10],[11]. In case where the force field is of good quality, i.e. points correctly to the heart edges, one may wish to force solution to respect the condition $\mathbf{f}(I+u)=0$ on the boundaries. The aim of this paper is to propose a singular perturbation of the Lamé system subject to a force field $\mathbf{f}$ extracted from image, which allows the condition $\mathbf{f}(I+u)=0$ to be fulfilled at convergence.

The paper is organized as follows. In section 2. the singular perturbation of the strain-stress relation is presented and it is proved that the asymptotic elastic model is well posed. In section 3. a finite element approximation is considered and numerical results are presented for a 2D cardiac MR image slice (mid-systole).

\section{Singular Perturbation of the Elastic Model}

The Deformable Elastic Template (DET) method introduced in [9] and later improved in [12], applied to a nonlinear model, makes use of a combination of the following items :

- A topological and geometric model of the object to be segmented.

- A constitutive equation (elasticity) defining its behavior under applied external image forces that pushes the model's interfaces towards the image edges.

The equilibrium of the model is obtained through the minimization of the following global energy functional :

$$
E(v)=E_{\text {elastic }}(v)+E_{\text {data }}(v)
$$


The model considered is that of a linear elastic body [3]. Its elastic energy reads :

$$
E_{\text {elastic }}=\frac{1}{2} \int_{\Omega_{0}} 2 \mu e(v): e(v)+\lambda \operatorname{div}^{2}(v) d x
$$

The object boundaries are submitted to a superficial gradient force field $\nabla g$. The expression of the external energy $E_{d a t a}$ is :

$$
E_{\text {data }}(v)=-\int_{\partial \Omega_{0}} g(v) d \gamma
$$

with $\partial \Omega_{0}$ the border of the object domain $\Omega_{0}$. Let $\Omega_{0}$ be the initial configuration of the elastic template, which is a bounded domain the boundary of which is of class $C^{2}$. The regularity of the boundary is needed for the solution to be regular [3].

We denote by $H^{1}\left(\Omega_{0}\right)$ the classical Sobolev space of functions in $L^{2}\left(\Omega_{0}\right)$ with a derivative in distributional sense in $L^{2}\left(\Omega_{0}\right)$

$$
H^{1}\left(\Omega_{0}\right)=\left\{\varphi \in L^{2}\left(\Omega_{0}\right) ; D \varphi \in L^{2}\left(\Omega_{0}\right)\right\}
$$

(see [3]) and we set $H=\left(L^{2}\left(\Omega_{0}\right)\right)^{3}$.

Let $R$ be the subspace of rigid motions, which is defined as the kernel of the strain tensor: $R=$ Ker $\epsilon$, set $\mathbb{H}=\left(H^{1}\left(\Omega_{0}\right) / R\right)^{3}$ the displacement space, equipped with the semi-norm $\|\epsilon(v)\|_{L^{2}\left(\Omega_{0}\right)}$ which, thanks to the Korn's inequality, is a Hilbert space. The following hypotheses for the field $\mathbf{f}$ are convenient for analyzing the asymptotic.

- H1: The function $\mathbf{f}$ is defined on a bounded domain $\mathcal{O} \supset \Omega_{0}$, and $\mathbf{f}$ is Lipschitz on $\mathcal{O}$ with values in $\mathbb{R}^{3}$. Furthermore, there exists $\mathbf{F}: \mathbb{R}^{3} \rightarrow \mathbb{R}^{+}$verifying $-D \mathbf{F}(x)=\mathbf{f}(x)$ with $\mathbf{f} \in R^{\perp}$.

The optimality conditions associated to minimizing $E(v)(2.1)$ on $\mathbb{H}$ read: find the displacement $u \in \mathbb{H}$ verifying:

$$
\left\{\begin{array}{l}
-\operatorname{div}(\sigma(u))=0 \quad \text { in } \Omega_{0} \\
\sigma(u) \cdot n=\mathbf{f}(I+u) \quad \text { on } \partial \Omega_{0}
\end{array}\right.
$$

Note that $u \neq 0 \Rightarrow \mathbf{f}(I+u) \neq 0$, so forces cannot be null for $u$ solution solution to (2.2).

\subsubsection{The Quasi-Static Lamé System}

The problem (2.2) is semi-linear, thus for computing an approximation with a finite element method (see for example ([9])), a quasi static or Picard fixed point strategies can be used: find $t \mapsto v(t)$ verifying:

$$
\left\{\begin{array}{l}
\frac{d}{d t} v(t)-\operatorname{div}(\sigma(v(t)))=0 \quad \text { in } \Omega_{0} \quad \text { for } 0<t \\
\sigma(v(t)) \cdot n=\mathbf{f}(I+v(t)) \quad \text { on } \partial \Omega_{0} \\
v(0)=0
\end{array}\right.
$$


or, for $w^{n}$ given, compute $w^{n+1}$ solution to:

$$
\left\{\begin{array}{l}
w^{n+1}-\operatorname{div}\left(\sigma\left(w^{n+1}\right)\right)=w^{n} \quad \text { in } \Omega_{0} \\
\sigma\left(w^{n+1}\right) \cdot n=\mathbf{f}\left(I+w^{n}\right) \quad \text { on } \partial \Omega_{0}
\end{array}\right.
$$

Then $u$ is defined by $u=\lim _{t \rightarrow \infty} v(t) ; u=\lim _{n \rightarrow \infty} w^{n}$. In the following, only the quasi-static Lamé system (2.3) will be investigated. For the fixed point strategy, the reader is referred to [10].

\subsection{Singular Perturbation Technique}

Let us introduce a function $\alpha$ satisfying:

- $\mathrm{H} 2$ : The function $\alpha: \mathbb{R}^{+} \rightarrow[0,1]$ is regular, integrable over $\mathbb{R}^{+}$, non increasing and bounded from below on any compact subset of $\mathbb{R}^{+}$with $\lim _{t \rightarrow \infty} \alpha(t)=0$. Moreover, $\sqrt{\alpha}$ is integrable over $\mathbb{R}^{+}$and verifies: $\lim _{t \rightarrow \infty} \int_{0}^{t} \sqrt{\alpha(s)} d s=0$.

We have:

Theorem 1. Assume hypotheses $H 1$ and $H 2$ to be satisfied. For any $T \in \mathbb{R}_{+}^{*}$ and for all $0<t<T$, the following problem

$$
\begin{cases}\frac{d}{d t} u(t)-\operatorname{div}(\alpha(t) \sigma(u(t))) & =0 \text { in } \Omega_{0} \\ \alpha(t) \sigma(u(t)) \cdot n & =\mathbf{f}(I+u(t)) \text { on } \partial \Omega_{0} \\ u(0)=0 & \text { in } \Omega_{0} .\end{cases}
$$

has a unique solution $u \in L^{2}\left(0, T ;\left(\left(H^{2}(\Omega)\right)^{3} \cap \mathbb{H}\right) \cap C^{0}(0, T ; \mathbb{H}) ; \frac{d}{d t} u \in L^{2}(0, T ; H)\right.$

Proof. The proof is classical in nature. A Hilbert basis of $\mathbb{H}$ is introduced, and then a Galerkin method is used to approximate the solution. Define the unbounded linear elasticity operator $A$ from $\left(L^{2}\left(\Omega_{0}\right)\right)^{3}$ into $\left(L^{2}\left(\Omega_{0}\right)\right)^{3}$ associated to Problem (2.2). Since the bilinear symmetric form $\bar{a}(\cdot, \cdot)$ associated to the linear elasticity operator $A$ :

$$
\bar{a}(v, w)=\int_{\Omega_{0}} 2 \mu e(v): e(w)+\lambda \operatorname{div} v \operatorname{div} w d x
$$

is $\mathbb{H}$-coercive due to Korn's inequality (see[8] Thm 3.5 p. 37 or [3] p. 298). Then for $h \in$ $\left(L^{2}\left(\Omega_{0}\right)\right)^{3} \cap R^{\perp}$ given, there exists a unique $v \in \mathbb{H}$ verifying

$$
\left\{\begin{array}{l}
-\operatorname{div}(\sigma(v))=h \quad \text { in } \Omega_{0} \\
\sigma(v) \cdot n=0 \quad \text { on } \partial \Omega_{0}
\end{array}\right.
$$

Moreover the regularity of the boundary provides, $v \in\left(H^{2}(\Omega)\right)^{3}$ ([7] p. 260). The embedding of $\left(H^{2}(\Omega)\right)^{3} \cap \mathbb{H}$ in $\mathbb{H}$ is compact since the domain $\Omega_{0}$ is bounded. The spectral theorem for selfadjoint compact operator works for $A^{-1}$ ([1] Thm VI.11 p. 97), the eigenvalues are non negative and form a sequence eventually converging towards zero. The associate eigenfunctions $\left\{w_{i}\right\}_{i=1}^{i=\infty}$ of 
$A^{-1}$ constitute an orthonormal basis of $\left(L^{2}\left(\Omega_{0}\right) / R\right)^{3}$ and of $\mathbb{H}$, and are orthogonal for the duality pairing

$$
\left(\left(w_{i}, w_{j}\right)\right)_{\mathbb{H}^{\prime}}=\left(A^{-1} w_{i}, w_{j}\right)_{L^{2}}=\left\{\begin{array}{c}
0 i \neq j ; \\
\frac{1}{\lambda_{i}} i=j
\end{array} .\right.
$$

Now let us give technical lemmas. For any $\varphi \in \mathbb{H}$ define the linear form $b(\varphi, \cdot): \mathbb{H} \rightarrow \mathbb{R}$ with:

$$
\forall \psi \in \mathbb{H}, \quad b(\varphi, \psi)=\int_{\partial \Omega_{0}} \mathbf{f}(I+\varphi) \psi d \gamma
$$

The time dependent $\mathbb{H}$-coercive bilinear form $a(\cdot, \cdot)$ is, for any $0<t \leq T$, defined by:

$$
a(v(t), w(t))=\alpha(t) \int_{\Omega_{0}} 2 \mu e(v(t)): e(w(t))+\lambda \operatorname{div} v(t) \operatorname{div} w(t) d x .
$$

Since the function $\alpha$ is bounded from below (see $\mathrm{H} 2$ ), by denoting $|v|_{1, \Omega_{0}}^{2}=\|\nabla v\|_{\left(L^{2}\left(\Omega_{0}\right)\right)^{3}}^{2}$ there exists $0<C$ verifying:

$$
C \min _{0 \leq s \leq t} \alpha(s)|v|_{1, \Omega_{0}}^{2} \leq \min _{0 \leq s \leq t} \alpha(s) \bar{a}(v, v) \leq a(v, v) \quad \forall v \in \mathbb{H}
$$

Lemma 2. For all $m \in \mathbb{N}$, there exists a unique family of functions $\left\{\varphi_{i}\right\}_{i=1}^{m}, \varphi_{i} \in C^{1}(0, \infty ; \mathbb{R})$ such that $u_{m}(t)=\sum_{i=1}^{m} \varphi_{i}(t) w_{i}$ is solution to:

$$
\left\{\begin{array}{l}
\left(\frac{d}{d t} u_{m}(t), w_{i}\right)+a\left(u_{m}(t), w_{i}\right)=b\left(u_{m}(t), w_{i}\right) \text { for } 1 \leq i \leq m \\
u_{m}(0)=0
\end{array}\right.
$$

Proof. Let $G_{i}\left(\varphi_{1}, \cdot \cdot, \varphi_{m}\right)$ be a real Lipschitzian function defined by:

$$
\begin{aligned}
& G_{i}\left(\varphi_{1}, \cdot \cdot, \varphi_{m}\right)=\int_{\partial \Omega_{0}} \mathbf{f}\left(I+\sum_{j=1}^{m} \varphi_{j}(t) w_{j}\right) w_{i} d \gamma \\
& \left|G_{i}\left(\varphi_{1}, \cdot \cdot, \varphi_{m}\right)-G_{i}\left(\psi_{1}, \cdot \cdot, \psi_{m}\right)\right| \leq \\
& K_{f} \max _{1 \leq j \leq m}\left|\varphi_{j}(t)-\psi_{j}(t)\right| \max _{1 \leq i, j \leq m} \int_{\partial \Omega_{0}}\left|w_{j}\right|\left|w_{i}\right| d \gamma
\end{aligned}
$$

We have:

$$
\left(w_{j}, w_{i}\right)=\delta_{i j} ; \quad a\left(w_{j}, w_{i}\right)=\delta_{i j} \text { for } 1 \leq i, j \leq m,
$$

thus Problem (2.7) is reduced to a diagonal system of differential equations:

$$
\left\{\begin{array}{l}
\frac{d}{d t} \varphi_{i}(t)+\lambda_{i} \alpha(t) \varphi_{i}(t)=G_{i}\left(\varphi_{1}(t), \cdot \cdot, \varphi_{m}(t)\right) \\
\varphi_{i}(t)=0 \text { for } 1 \leq i \leq m .
\end{array}\right.
$$

Theorem VII.3 p. 104 in [1] applies which proves the stated result.

Now we prove some a priori estimates for $u_{m}$. 
Lemma 3. There exit two constants $0<C_{2}$ and $0<C_{3}$ irrespective of $m$ and $t$ such that the following estimates hold true:

$$
\begin{aligned}
& \max _{0 \leq s \leq t} \int_{0}^{s} \frac{1}{2}\left\|\frac{d}{d \tau} u_{m}(\tau)\right\|_{L^{2}\left(\Omega_{0}\right)}^{2} d \tau \leq C_{2} ; \quad \max _{0 \leq s \leq t}\left\|\sqrt{\alpha(s)} u_{m}(s)\right\|_{1, \Omega_{0}}^{2} \leq C_{2} ; \\
& \int_{0}^{t}\left\|\alpha(\tau) u_{m}(\tau)\right\|_{H^{2}\left(\Omega_{0}\right)}^{2} d \tau \leq C_{3} .
\end{aligned}
$$

Proof. Substitute the test function by $\frac{d}{d t} u_{m}$ in (2.7), and integrate from 0 to $s$ and over $\Omega_{0}$. Accordingly the gradient structure of $f$, we have:

$$
\int_{\partial \Omega_{0}} \mathbf{f}\left(I+u_{m}(\tau)\right) \frac{d}{d \tau} u_{m}(\tau) d \gamma,=-\int_{\partial \Omega_{0}} \frac{d}{d \tau} \mathbf{F}(I+u(\tau)) d \gamma
$$

After some classical calculations, we get:

$$
\left.\left\|\frac{d}{d \tau} u_{m}(\tau)\right\|_{L^{2}\left(\Omega_{0}\right)}^{2}+\frac{\alpha(\tau)}{2} \frac{d}{d \tau} \bar{a}\left(u_{m}(\tau), u_{m}(\tau)\right)=-\int_{\partial \Omega_{0}} \frac{d}{d \tau} \mathbf{F}\left(I+u_{m}(\tau)\right)\right) d \gamma
$$

For all $0<s<t$, by integrating by parts the second term, we get:

$$
\begin{array}{r}
\int_{0}^{s}\left\|\frac{d}{d \tau} u_{m}(\tau)\right\|_{L^{2}\left(\Omega_{0}\right)}^{2} d \tau-\int_{0}^{s} \frac{\alpha^{\prime}(\tau)}{2} \bar{a}\left(u_{m}(\tau), u_{m}(\tau)\right) d \tau+\frac{\alpha(s)}{2} \bar{a}\left(u_{m}(s), u_{m}(s)\right)+ \\
\int_{\partial \Omega_{0}} \mathbf{F}\left(I+u_{m}(s)\right) d \gamma=\int_{\partial \Omega_{0}} \mathbf{F}(x) d \gamma
\end{array}
$$

So

$$
\int_{0}^{s}\left\|\frac{d}{d \tau} u_{m}(\tau)\right\|_{L^{2}\left(\Omega_{0}\right)}^{2} d \tau+\frac{C}{2}\left\|\sqrt{\alpha(s)} u_{m}(s)\right\|_{1, \Omega_{0}}^{2}+\int_{\partial \Omega_{0}} \mathbf{F}\left(I+u_{m}(s)\right) d \gamma=\int_{\partial \Omega_{0}} \mathbf{F}(x) d \gamma .
$$

The function $|F|$ is bounded by $M_{F}$, thus we get the two first inequalities with $C_{2}=M_{F}\left|\partial \Omega_{0}\right|$.

Lemma 2 tells us $u_{m}$ is a solution of Problem (2.5) in a finite dimensional vector space. Multiply the first equation of (2.5) with $-\operatorname{div}\left(\alpha(t) \sigma\left(u_{m}(t)\right)\right)$ and integrate over $\Omega_{0}$ and with respect to time. Holder inequality provides:

$$
\int_{0}^{s}\left\|\operatorname{div}\left(\alpha(\tau) \sigma\left(u_{m}(\tau)\right)\right)\right\|_{L^{2}\left(\Omega_{0}\right)}^{2} d \tau \leq 2 \int_{0}^{s}\left\|\frac{d}{d \tau} u_{m}(\tau)\right\|_{L^{2}\left(\Omega_{0}\right)}^{2} d \tau \leq C_{2}
$$

The linear elasticity operator $A$ is uniformly elliptic ([7] p. 260), thus we have :

$$
\int_{0}^{s}\left\|\alpha(\tau) u_{m}(\tau)\right\|_{H^{2}\left(\Omega_{0}\right)}^{2} d \tau-\beta \int_{0}^{s}\left\|\alpha(\tau) u_{m}(\tau)\right\|_{H^{1}\left(\Omega_{0}\right)}^{2} d \tau \leq \int_{0}^{s}\left\|\operatorname{div}\left(\alpha(\tau) \sigma\left(u_{m}(\tau)\right)\right)\right\|_{L^{2}\left(\Omega_{0}\right)}^{2} d \tau .
$$

Korn's inequality and the second estimate in (2.11) allows us to establish the last inequality. 
From the Lemmas proved before we deduce the following. There exist $K_{1}$ a bounded subset of $L_{l o c}^{2}(0, \infty ; H), K_{2}$ a bounded subset of $L^{\infty}(0, \infty ; \mathbb{H})$ and $K_{3}$ a bounded subset of $L_{\text {loc }}^{2}\left(0, \infty ;\left(\left(H^{2}\left(\Omega_{0}\right)\right)^{3} \cap \mathbb{H}\right)\right)$ such that:

$$
\left\{\frac{d}{d t} u_{m}\right\}_{m=1}^{\infty} \subset K_{1} ; \quad\left\{u_{m}\right\}_{m=1}^{\infty} \subset K_{2} ; \quad\left\{u_{m}\right\}_{m=1}^{\infty} \subset K_{3} .
$$

The weak sequential compactness of the unit ball of a reflexive Banach space allows us to take the limit when $m$ goes to infinity.

Lemma 4. There exists

$$
u \in L^{\infty}(0, \infty ; H) \cap L^{\infty}(0, \infty ; \mathbb{H}) \cap L_{l o c}^{2}\left(0, \infty ;\left(H^{2}\left(\Omega_{0}\right)\right)^{3} \cap \mathbb{H}\right) \cap C^{0}(0, \infty ; \mathbb{H})
$$

such that, up to a subsequence, for every $0<T$,

$$
\begin{aligned}
& \int_{0}^{T}\left(u_{m}(s), \varphi(s)\right)_{H} d s \rightarrow \int_{0}^{T}(u(s), \varphi(s))_{H} d s \quad \forall \varphi \in L^{1}(0, T ; H) ; \\
& \int_{0}^{T}\left(u_{m}(s), \varphi(s)\right)_{\mathbb{H}^{\prime} \mathbb{H}} d s \rightarrow \int_{0}^{T}\left(u_{m}(s), \varphi(s)\right)_{\mathbb{H}^{\prime} \mathbb{H}} d s \quad \forall \varphi \in L^{1}(0, T ; \mathbb{H}) ; \\
& \int_{0}^{T} a\left(u_{m}(s), \varphi(s)\right) d s \rightarrow \int_{0}^{T} a(u(s), \varphi(s)) d s \quad \forall \varphi \in L^{1}(0, T ; \mathbb{H}) ; \\
& \frac{d}{d t}\left(u_{m}, \varphi\right)_{H} \rightarrow \frac{d}{d t}(u, \varphi)_{H} \quad \forall \varphi \in H \text { in } \mathcal{D}^{\prime}(0, \infty) .
\end{aligned}
$$

The last step is to prove that $u$ is a solution to Problem (2.5). So let us take the limit in the variational formulation (2.7). Since $\Omega_{0}$ is bounded, the embedding of $H^{2}\left(\Omega_{0}\right)$ onto $H^{1}\left(\Omega_{0}\right)$ is compact. We deduce that the following embedding is compact (see for example [13])

$$
\left\{\varphi \in L^{2}\left(0, T ;\left(H^{2}\left(\Omega_{0}\right)^{3} \cap \mathbb{H}\right)\right), \frac{d}{d t} \varphi \in L^{2}(0, T ; H)\right\} \subset C^{0}(0, T ; \mathbb{H}) \subset C^{0}\left(0, T ;\left(L^{2}\left(\partial \Omega_{0}\right)^{3}\right) .\right.
$$

Thus, for every $0<t<T, u_{m}(t, x) \rightarrow u(t, x)$ for almost every $x \in \partial \Omega_{0}$, and since $\mathbf{f}$ is continuous, up to a subsequence, we have $\mathbf{f}\left(x+u_{m}(t, x)\right) \rightarrow \mathbf{f}(x+u(t, x))$ for every $t$ and for every $x \in \partial \Omega_{0}$. The Lebesgue's dominated convergence Theorem applies and we get:

$$
\lim _{m \rightarrow \infty} b\left(u_{m}, \varphi\right) \rightarrow b(u, \varphi) \quad \forall \varphi \in\left(L^{2}\left(\partial \Omega_{0}\right)^{3}\right) .
$$

We have proved the existence of a solution for Problem 2.5. Uniqueness is shown in a classical way [13]. Take the difference of two solutions, and since the function $\mathbf{f}$ is bounded, we get an a priori estimate for the $L^{2}$ norm of the difference and for its time derivative. The Gronwall's Lemma applies and the uniqueness is proved since the two solutions have the same initial condition.

For Problem (2.5), the asymptotic behavior with respect to time is given in the next theorem.

Theorem 5. Assume hypotheses $H 1$ and $H 2$ to be satisfied. Then, when $t$ goes to infinity, $u(t)$ solution to (2.5) converges towards $\bar{u} \in H$ and

$$
\lim _{t \rightarrow+\infty} \mathbf{f}(I+u(t))=0 \operatorname{in}\left(H^{-1 / 2}\left(\partial \Omega_{0}\right)\right)^{3} .
$$


Proof. Arguing in the same way as in the proof of Lemma 3, we have two constants $C_{4}$ and $C_{5}$ irrespective of time $t$ such that:

$$
\left\|\frac{d u(s)}{d s}\right\|_{\left(L^{2}\left(0, t ;\left(L^{2}\left(\Omega_{0}\right)\right)\right)\right)^{3}}^{2} \leq C_{4} ; \quad\|\sqrt{\alpha(t)} u(t)\|_{\left(H^{1}\left(\Omega_{0}\right)\right)^{3}}^{2} \leq C_{5} .
$$

Whatever $\varphi \in\left(H_{0}^{1}\left(\Omega_{0}\right)\right)^{3}$ is we deduce from Problem (2.5) that

$$
\left|\int_{\Omega_{0}} \frac{d u(t)}{d t} \varphi d x\right| \leq \sqrt{\alpha(t)}|\bar{a}(\sqrt{\alpha(t)} u(t), \varphi)| \leq \sqrt{\alpha(t)} \sqrt{C_{5}}\|\varphi\|_{\left(H^{1}\left(\Omega_{0}\right)\right)^{3}}
$$

We conclude that up to a zero measure subset, $\frac{d}{d t} u(t)$ goes to zero in $\left(H^{1}\left(\Omega_{0}\right)^{\prime}\right)^{3}$ when time goes to infinity. Moreover, by considering a variational formulation of Problem (2.5), we have for all $\varphi \in\left(H^{1}\left(\Omega_{0}\right)\right)^{3}$ :

$$
\left|\int_{\partial \Omega_{0}} \mathbf{f}(I+u(t)) \varphi d \xi\right| \leq\left|\int_{\Omega_{0}} \frac{d}{d t} u(t) \varphi d x\right|+\sqrt{\alpha(t)}|\bar{a}(\sqrt{\alpha(t)} u(t), \varphi)| .
$$

Up to a zero measure subset, the right hand side of the previous expression goes to zero when $t$ goes to infinity. Since $u(t) \in C^{0}(0, \infty ; \mathbb{H})$, and since $\mathbf{f}$ is continuous, we conclude that

$$
\lim _{t \rightarrow \infty} \mathbf{f}(I+u(t))=0 \text { in }\left(H^{-1 / 2}\left(\partial \Omega_{0}\right)\right)^{3} .
$$

The function $u \in C^{0}(0, \infty ; \mathbb{H})$ thus belongs to $C^{0}(0, \infty ; H)$. Set $\bar{u}=\lim _{t \rightarrow+\infty} \frac{1}{t} \int_{0}^{t} u(s) d s$, then let us prove that: $\lim _{t \rightarrow+\infty} u(t)=\bar{u}$ in $\left(H^{-1}\left(\Omega_{0}\right)\right)^{3}$.

We have:

$$
\begin{aligned}
& \quad \sup \|_{H_{0}^{1}\left(\Omega_{0}\right)}=1 \int_{\Omega_{0}}\left(\frac{1}{t} \int_{0}^{t} u(t)-u(s) d s\right) \varphi d x \\
& =\|\varphi\|_{H_{0}^{1}\left(\Omega_{0}\right)}=1 \int_{\Omega_{0}}\left(\frac{1}{t} \int_{0}^{t} \int_{s}^{t} \frac{1}{d \tau} u(\tau) d \tau d s\right) \varphi d x \leq \sqrt{C_{5}} \frac{1}{t} \int_{0}^{t} \int_{0}^{t} \sqrt{\alpha(\tau)} d \tau d s
\end{aligned}
$$

Hypothesis $\mathrm{H} 2$ allows us to conclude.

Now, a compactness related argument is invoked for proving the convergence for almost every $t$ in $\left(L^{2}\left(\Omega_{0}\right)\right)^{3}$. Define $\varphi_{n}(t)=\left\{\begin{array}{l}1 ; 0 \leq t \leq n \\ n+1-t ; n \leq t \leq n+1 \\ 0 ; \text { elsewhere }\end{array}\right.$ and $u_{n}(t)=\varphi_{n}(t) u(t)$.

Let $\epsilon>0$ be fixed, there exists $R_{\epsilon}$ such that $\forall t>R_{\epsilon}\|u(t)-\bar{u}\|_{\left(H^{-1}\left(\Omega_{0}\right)\right)^{3}}<\epsilon$. Let $T>R_{\epsilon}$, $\left\{u_{n}\right\}_{n \in \mathbb{N}}$ is bounded in $L^{2}(0, T ; \mathbb{H})$ and $\frac{d u_{n}}{d t}$ is bounded in $L^{2}(0, T ; H)$. Theorem 1 in [14] applies, and there exists $\underline{u} \in L^{2}(0, T ; H)$ and a subsequence $\left\{n_{p}\right\}_{p \in \mathbb{N}}$ such that $u_{n_{p}} \rightarrow \underline{u}$ in $L^{2}(0, T ; H)$ and, up to a nul set of $(0, T) u_{n_{p}}(t) \rightarrow \underline{u}(t)$ in $\left(L^{2}\left(\Omega_{0}\right)\right)^{3}$. Notice that for $n_{p}>E\left(R_{\epsilon}\right)+1$ we have $u_{n_{p}}(t)=u(t)$. For almost every $t>R_{\epsilon}$, we have

$$
\|u(t)-\underline{u}(t)\|_{\left(H^{-1}\left(\Omega_{0}\right)\right)^{3}}^{2} \leq\|u(t)-\underline{u}(t)\|_{\left(L^{2}\left(\Omega_{0}\right)\right)^{3}}^{2} \leq \epsilon .
$$

We deduce that for almost every $t, \lim _{t \rightarrow \infty} u(t)=\bar{u}$ in $H$. 


\section{Finite Element Approximation}

We implemented the previously described technique using a first order Lagrange finite element method for discretizing the spatial functions, and a simple Euler scheme for time integration. Finite elements are triangles, and the initial mesh is a uniform ring. The finite dimensional space is denoted by $V_{h}$. In our implementation, we used $\alpha(t)=e^{-\beta t}$. Let $W_{h}$ denote the trace on $\partial \Omega_{0}$ of elements of $V_{h}$. The $L^{2}\left(\partial \Omega_{0}\right)$ projector into $W_{h}$ is denoted by $\Pi_{h}$. The finite element formulation of problem (2.5) reads:

$$
\frac{\mathbf{U}^{n}-\mathbf{U}^{n-1}}{\Delta t}+e^{-\beta t_{n}} \mathbf{K} \mathbf{U}^{n}=\mathbf{F}\left(\mathbf{U}^{n-1}\right)
$$

or

$$
\left(\Delta t e^{-\beta t_{n}} \mathbf{K}+\mathbf{I}\right) \mathbf{U}^{n}=\Delta t \mathbf{F}\left(\mathbf{U}^{n-1}\right)+\mathbf{U}^{n-1}
$$

where $\mathbf{F}$ is the vector of forces, $\mathbf{K}$ is the stiffness matrix corresponding to the elasticity operator $a(\cdot, \cdot), \Delta t$ is the integration time step and $\mathbf{U}$ is the displacement vector (displacement of mesh node points). The parameter $\beta$ should be chosen so that the exponential varies slowly compared to $\mathbf{F}(\mathbf{U})$, and thus depends on the Lipschitz constant of the force field.

In order to test the model on experimental data, we used the free 4D heart database [4]. This database consists of cardiac MR images of 18 patients, together with two expert segmentations at end-diastole and end-systole. A short axis slice sequence, corresponding to a median heart level, has been extracted from one patient set.

The initial shape of the model used to extract the left ventricular (LV) contours from the 2D image sequences was simply a ring. Indeed, the shape of a short axis slice of the myocardium in healthy patients is very close to a perfect ring. This ring was meshed with a very simple method : first divide the ring into quadrangles using sectors and concentric rings, then divide each quadrangle into two triangles. Better meshing methods could of course be used, but the triangles generated by our simple method proved good enough for this application.

Let us denote by $u_{h}(t)$ the solution of the finite element approximation problem. Arguing in the same way as in the previous section, and accounting for $V_{h}$ is a finite dimension space, we have:

$$
\begin{aligned}
& \Pi_{h} \mathbf{f}\left(I+u_{h}(t)\right) \rightarrow{ }_{t \rightarrow \infty} 0 \text { in } H^{1 / 2}\left(\partial \Omega_{0}\right) \text { thus in } L^{2}\left(\partial \Omega_{0}\right) ; \\
& \left.u_{h}(t)\right) \rightarrow{ }_{t \rightarrow \infty} \bar{u}_{h} \text { in } C^{0}\left(0, \infty ; H^{1}\left(\Omega_{0}\right)\right) \text { thus in } C^{0}\left(0, \infty ; H^{1 / 2}\left(\partial \Omega_{0}\right)\right) .
\end{aligned}
$$

Since $\mathbf{f}$ is continuous, we deduce that: $\Pi_{h} \mathbf{f}\left(I+\bar{u}_{h}\right)=0$.

Since the method ensures at the mesh node points that $\mathbf{f}\left(I+\bar{u}_{h}\right)=0$ at convergence, we are guaranteed that the contours of the model will match the zeros of the force fields, that is to say, the contours extracted by a low-level preprocessing method. This is very interesting when confidence in these contours is high but can be a problem when they are noisy.

Figure 2 shows the effect of the proposed scheme on an instant of the real MR sequence. The pink mesh is the model. It is set to be translucent so that correspondence between model and image contours can be visualized. The proposed scheme allows to perfectly match borders at the expense of contour regularity. It should thus be used only when the pre-segmentation is very good and the model is used mostly for motion estimation. 


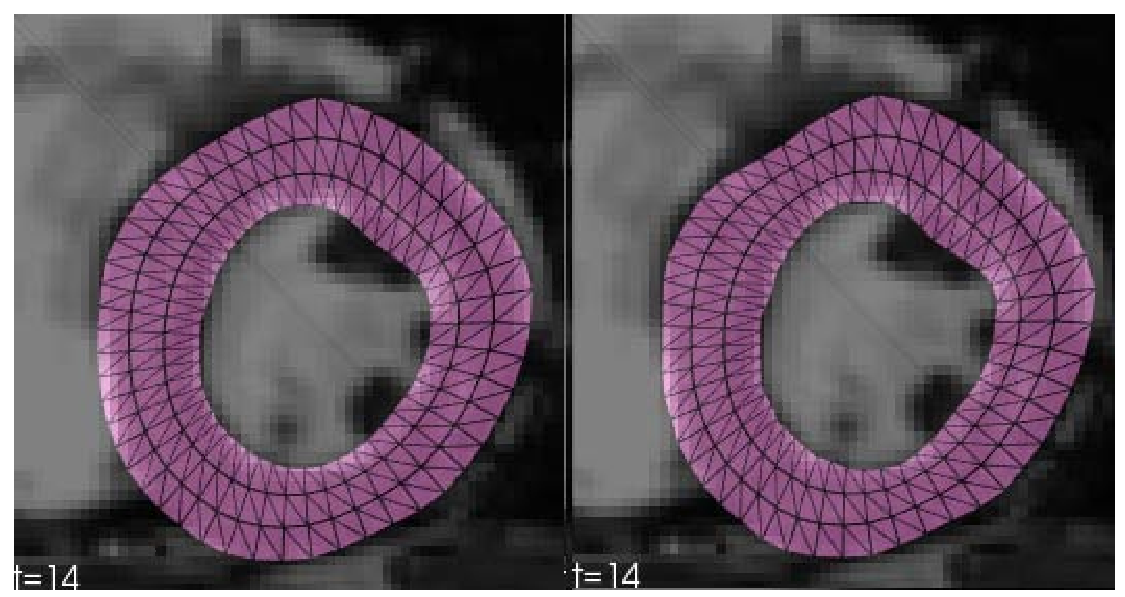

Figure 2: Effect of the proposed scheme: on the left, normal scheme; on the right, proposed scheme. Notice that the proposed scheme follows borders more accurately, at the expense of contour regularity.

\section{References}

[1] H. Brézis. Analyse fonctionnelle. Masson, Paris 1985.

[2] C, Xu, J. L. Prince. Snakes, shapes, and gradient vector flow. IEEE Transactions on Image Processing, 7 1998, 359-369.

[3] P.G. Ciarlet. Mathematical elasticity. North-Holland 1993.

[4] J. Cousty, L. Najman, M. Couprie, S. Clément-Guinaudeau, T. Goissen, J. Garot. Automated, accurate and fast segmentation of $4 D$ cardiac MR images. Functional Imaging and Modeling of the Heart (FIMH), LNCS, Springer, (2007), No. 4466, 474-483.

[5] B. Faugeras, J. Pousin. Variational asymptotic derivation of an elastic model arising from the problem of $3 D$ automatic segmentation of cardiac images. Analysis and Applications, 2 (2004), No. 4, 1-33.

[6] F. Krasucki, S. Lenci. Yield design of bonded joints. Eur. J. Mech. A Solids, 19 (2000), No. 4, 649-667.

[7] J. Necas. Les méthodes directes en théorie des équations elliptiques. Masson, Paris, 1967.

[8] O.A. Oleinik, A.S. Shamaev, G.A. Yosifian. Mathematical problems in elasticity and homogenization. Studies in Mathematics and its applications, Noth-Holland 1992.

[9] Q.C. Pham, F. Vincent, P. Clarysse, P. Croisille, I.E. Magnin. A FEM-based deformable model for the $3 D$ segmentation and tracking of the heart in cardiac MRI., Proceedings of the 2nd 
International Symposium on Image and Signal Processing and Analysis, ISPA (2001), 250254.

[10] M. Picq J. Pousin, Y. Rouchdy, A linear 3D elastic segmentation model for vector fields. Application to the heart segmentation in MRI. Journal Of Mathematical Imaging and Vision, 27 (2007), No. 3, 241-255.

[11] P. Pebay, T. Baker, J. Pousin. Dynamic meshing for finite element based segmentation of cardiac imagery. Fifth World Congress on Computational Mechanics, (2002).

[12] Y. Rouchdy, J. Pousin, J. Schaerer, P. Clarysse. A nonlinear elastic deformable template for soft structure segmentation. Application to heart segmentation in MRI. J. Inverse Problems, 23 (2007), No. 3, 1017-1035.

[13] L. Tartar. Topics in nonlinear analysis. Publications Mathématiques d'Orsay, 1978.

[14] J. Simon. Compact Sets in the Space $L^{p}(O, T ; B)$. Ann. Math. Pura Appl., (IV) Vol. CXLVI (1987), 65-96. 